\title{
Fraxetin Induces Heme Oxygenase-1 Expression by Activation of Akt/Nrf2 or AMP-activated Protein Kinase $\alpha /$ Nrf2 Pathway in HaCaT Cells
}

\author{
Juthika Kundu*'**, In Gyeong Chae*, Kyung-Soo Chun \\ College of Pharmacy, Keimyung University, Daegu, Korea
}

\begin{abstract}
Background: Fraxetin (7,8-dihydroxy-6-methoxy coumarin), a coumarin derivative, has been reported to possess antioxidative, antiinflammatory and neuroprotective effects. A number of recent observations suggest that the induction of heme oxygenase-1 (HO-1) inhibits inflammation and tumorigenesis. In the present study, we determined the effect of fraxetin on HO-1 expression in HaCaT human keratinocytes and investigated its underlying molecular mechanisms.

Methods: Reverse transcriptase-PCR and Western blot analysis were performed to detect HO-1 mRNA and protein expression, respectively. Cell viability was measured by the MTS test. The induction of intracellular reactive oxygen species (ROS) by fraxetin was evaluated by 2',7'-dichlorofluorescin diacetate staining.

Results: Fraxetin upregulated mRNA and protein expression of HO-1. Incubation with fraxetin induced the localization of nuclear factor-erythroid-2-related factor-2 (Nrf2) in the nucleus and increased the antioxidant response element-reporter gene activity. Fraxetin also induced the phosphorylation of Akt and AMP-activated protein kinase (AMPK) $\alpha$ and diminished the expression of phosphatase and tensin homolog, a negative regulator of Akt. Pharmacological inhibition of Akt and AMPK $\alpha$ abrogated fraxetin-induced expression of HO-1 and nuclear localization of Nrf2. Furthermore, fraxetin generated ROS in a concentration-dependent manner.
\end{abstract}

Conclusions: Fraxetin induces HO-1 expression through activation of Akt/Nrf2 or AMPKa/Nrf2 pathway in $\mathrm{HaCaT}$ cells.

(J Cancer Prev 2016;21:135-143)

Key Words: Fraxetin, Keratinocytes, Heme oxygenase-1, Nrf2

\section{INTRODUCTION}

Oxidative stress is known as an imbalance between the production of free radicals and their elimination by protective mechanisms. ${ }^{1}$ Reactive oxygen species (ROS), such as superoxide anions, hydroxyl radicals and hydrogen peroxide, are generated by ultraviolet (UV) light, cigarette smoke, or aging. Excessive production of ROS causes oxidative damage of cellular macromolecules, such as DNA, proteins and lipids, thereby inducing neoplastic transformation of cells. ${ }^{2}$ Skin is constantly exposed to oxidative stress from exogenous and endogenous sources. UV-generated ROS in the skin develop oxidative stress when formation of ROS exceeds the antioxidant defense ability. Therefore, antioxidant systems are important to maintain cellular homeostasis and the cellular redox system consists of a series of enzymatic and nonenzymatic antioxidants in skin. ${ }^{3}$

Heme oxygenase-1 (HO-1) is a representative cytoprotective enzyme that catalyzes the rate limiting steps in heme degradation. Multiple lines of evidence have revealed that HO-1 attenuates skin inflammation ${ }^{4}$ and atopic dermatitis-like skin lesions. ${ }^{5}$ Topical treatment of mouse skin with hemin, a chemical inducer of HO-1, reduces 12-O-tetradecanoylphorbol-13-acetate (TPA)-induced tumor promotion. ${ }^{6,7}$ Moreover, wild type mice having endogenous HO-1 are less susceptible to TPA-induced skin

Received August 28, 2016, Revised September 6, 2016, Accepted September 7, 2016

Correspondence to: Kyung-Soo Chun

College of Pharmacy, Keimyung University, 1095 Dalgubeol-daero, Dalseo-gu, Daegu 42601, Korea

Tel: +82-53-580-6647, Fax: +82-53-580-6645, E-mail: chunks@kmu.ac.kr, ORCID: Kyung-Soo Chun, http://orcid.org/0000-0001-9933-2897

*These authors contributed equally to this work (paper) as co-first authors.

**Current affiliation: LiKa Shing Institute of Virology, University of Alberta, Edmonton, AB, Canada.

Copyright (C) 2016 Korean Society of Cancer Prevention

(c) This is an Open Access article distributed under the terms of the Creative Commons Attribution Non-Commercial License (http://creativecommons.org/licenses/by-nc/4.0) which permits unrestricted non-commercial use, distribution, and reproduction in any medium, provided the original work is properly cited. 
tumor formation in the early stage of papillomagenesis as compared to HO-1 knock-out mice. ${ }^{8}$ Shin et al. ${ }^{9}$ reported that zerumbone, a sesquiterpene derived from tropical ginger, induces HO-1 expression through activation of nuclear factor erythroid-2-related factor-2 (Nrf2) in JB6 cells and mouse skin, which may partly account for its previously reported inhibitory effects on mouse skin carcinogenesis. Thus, fortification of skin tissue with elevated HO-1 confers protection against cancer.

The expression of HO-1 is known to be regulated through the activation of a redox-sensitive transcription factor, Nrf2. ${ }^{10,11}$ Under basal conditions, Nrf2 is constitutively sequestered in the cytoplasm by its repressor Kelch-like ECH-associated protein (Keap1), which causes proteasomal degradation of Nrf2. ${ }^{12-15}$ However, under condition of oxidative stress, Keap1 undergoes oxidative or covalent modifications of cysteine residues, ${ }^{16}$ thereby allowing stabilization of Nrf2 through its dissociation from Keap1. Thus, Nrf2 migrates to the nucleus and activates the antioxidant response element (ARE) within the ho-1 gene promoter region. ${ }^{17,18}$ Besides oxidative modification of Keap1 cysteine thiols, dissociation of Nrf2 from Keap 1 can be promoted upon phosphorylation at specific serine or threonine residues of Nrf2 by kinases, such as phosphatidylionositol-3-kinase (PI3K)/Akt, ${ }^{10}$ AMP-activated protein kinase (AMPK), ${ }^{20}$ mitogen-activated protein (MAP) kinases, ${ }^{21.22}$ and protein kinase $\mathrm{C}^{23}$ In our previous study, we demonstrated that thymoquinone, an active constituent of Nigella sativa, increases HO-1 expression by activating Nrf2 via ROS-mediated phosphorylation of Akt and AMPK $\alpha$ in human keratinocytes (HaCaT). ${ }^{24}$

Fraxinus rhynchophylla, belonging to the Oleaceae family, possesses several biological activities, including anti-oxidative, ${ }^{25}$ anti-inflammatory ${ }^{26}$ and anti-toxoplasmosis ${ }^{27}$ effects. Fraxetin (6-metoxy-7,8-dihydroxycoumarin), a major dihydroxycoumarin found in bark of $E$ rhynchophylla, has been shown to have strong anti-oxidant, $^{28-30}$ anti-inflammatory, ${ }^{31}$ neuroprotective ${ }^{32}$ and anti-tumor ${ }^{33}$ properties (Fig. 1A). In this study, we investigated whether fraxetin could induce HO-1 expression in $\mathrm{HaCaT}$ cells and elucidated its underlying mechanisms with special focus on its role in modulating Nrf2 signaling.

\section{MATERIALS AND METHODS}

\section{Chemicals and reagents}

All chemicals were obtained from Sigma Chemical (St. Louis, MO, USA) unless otherwise indicated. Cell culture reagents were purchased from Gibco BRL (Rockville, MD, USA). Antibodies against HO-1, p-Akt, Akt, AMPK $\alpha$, p-AMPK $\alpha$ and Akt inhibitor
LY294002 were purchased from Cell Signaling Technology (Beverly, MA, USA). Primary antibody against each of Nrf2 and phosphatase and tensin homolog and horse-raddish peroxidase-conjugated secondary antibodies were purchased from SantaCruz Biotechnology (SantaCruz, CA, USA). The 2',7'dichlorofluorescin diacetate (DCF-DA) was purchased from Invitrogen (Carlsbad, CA, USA). Hank's balanced salt solution (HBSS) was purchased from Meditech (Herndon, VA, USA).

\section{Cell culture and treatment}

$\mathrm{HaCaT}$ cells were obtained from the American Type Culture Collection (Rockville, MD, USA). Cell types were maintained in Dulbecco's modified Eagle's medium (DMEM) supplemented with $10 \%$ FBS containing $100 \mathrm{U} / \mathrm{mL}$ of penicillin and $100 \mu \mathrm{g} / \mathrm{mL}$ of streptomycin and $5 \% \mathrm{CO}_{2}$ at $37^{\circ} \mathrm{C}$. For all experiments, early passage cells were grown to $80 \%$ to $90 \%$ confluence. All chemicals were dissolved in dimethyl sulfoxide (DMSO) and the final DMSO concentration was less than $0.1 \%$.

\section{Cytotoxicity assay}

Cytotoxicity effect of fraxetin against $\mathrm{HaCaT}$ cells was measured by using a solution of tetrazolium compound (MTS) (Promega, Madison, WI, USA). Briefly, the cells were plated in 96-well culture plates at a density of $2 \times 10^{3}$ cells/well in DMEM and allowed to attach for 24 hours. After incubation, the medium was discarded and replaced with $100 \mu \mathrm{L}$ of new medium containing various concentrations of fraxetin. After 24 hours or 48 hours of culture, $20 \mu \mathrm{L}$ MTS reagent was added to each well, followed by 1 hour incubation. The optical density was measured at $550 \mathrm{~nm}$ in a microplate reader (Tecan Trading AG, Männedorf, Switzerland). Cell viabilities were presented as the percentage of the optical density of samples to that of the control.

\section{Western blot analysis}

Cells were harvested by scraping the cells from cultured dishes using a cell scraper. Cellular lysates were prepared using RIPA cell lysis buffer. The cells were disrupted and extracted at $4^{\circ} \mathrm{C}$ for 30 minutes. After centrifugation at $16,000 \times g$ for 15 minutes, the supernatant was obtained as the cell lysate. Protein concentrations were measured using a protein assay kit (Pierce Biotechnology, Rockford, IL, USA). Cellular proteins ( $30 \mu \mathrm{g}$ ) were subjected to $10 \%$ SDS-PAGE. The resolved proteins were transferred to an Immobilon-P-membrane and allowed to react with a specific antibody. The detection of specific proteins was carried out by Super-signal pico-chemiluminescent substrate or dura-luminol substrate (Thermo Scientific, Waltham, MA, USA) accor- 
A

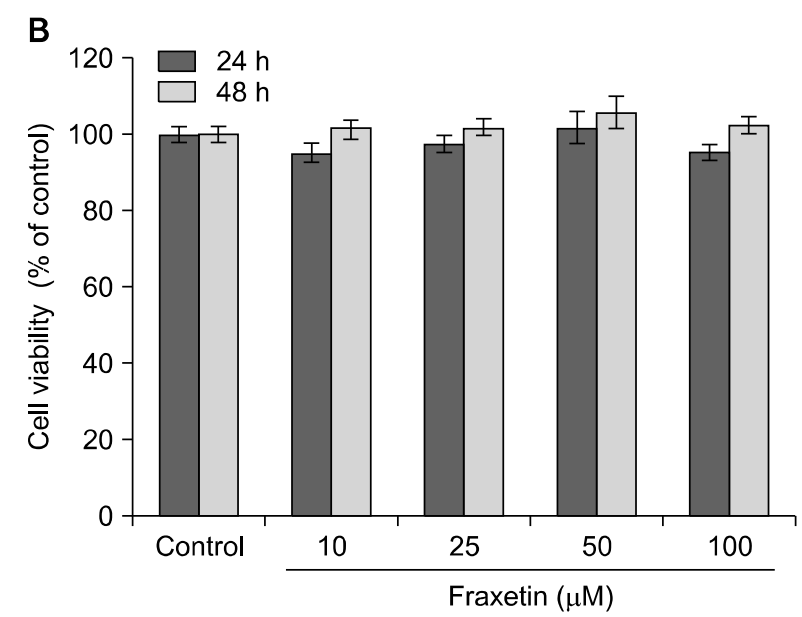

C<smiles>COc1cc2ccc(=O)oc2c(O)c1O</smiles>

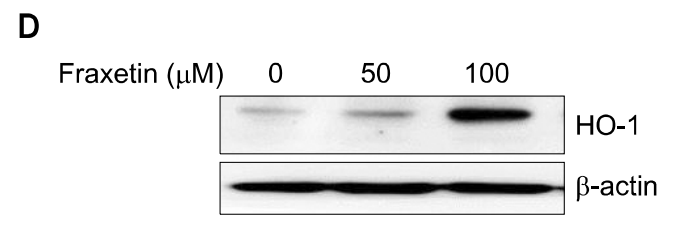

E

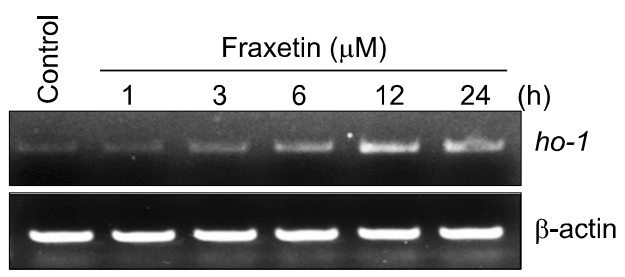

Fraxetin $(\mu \mathrm{M}) \quad 0 \quad 50 \quad 100$

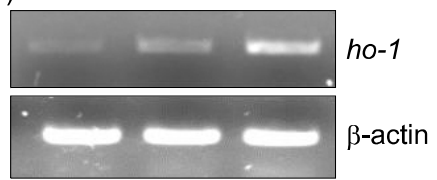

$\beta$-actin
$\mathbf{F}$

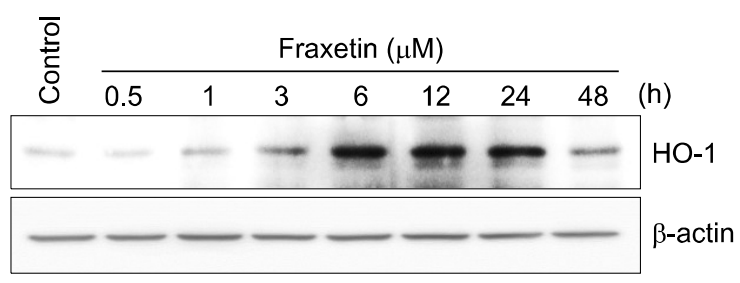

Figure 1. Effect of fraxetin on heme oxygenase-1 expression in HaCaT cells. (A) Chemical structure of fraxetin. (B) HaCaT cells were treated with indicated concentration of fraxetin for 24 hours and 48 hours, and cell viability was evaluated by the MTS assay. The results are presented as means $\pm \mathrm{SD}(\mathrm{n}=3)$. (C, D) Cell were treated with indicated concentration of fraxetin for 6 hours. (E, F) Cell were treated with fraxetin $(100 \mu \mathrm{M})$ for indicated time. (C, E) Expression of heme oxygenase-1 (HO-1) mRNA (ho-1) was detected by reverse transcriptase-PCR and (D, F) protein level of HO-1 was determined by Western blot analysis.

ding to manufacturer's instruction and visualized with imagequant LAS 4000 (Fujifilm Life Science, Tokyo, Japan). Loading differences were normalized using anti- $\beta$-actin antibody.

\section{Reverse transcriptase-PCR}

Total RNA was isolated using TRIzol (Invitrogen). Total RNA (1 $\mu \mathrm{g}$ ) was used for the complementary DNA synthesis using random primers. PCR conditions for ho-1 was as follows: ho-1, 35 cycles for $95^{\circ} \mathrm{C}$ for 2 minutes, $54.2^{\circ} \mathrm{C}$ for 40 seconds and $72^{\circ} \mathrm{C}$ for 1 minute. All primers were synthesized from Bioneer (Seoul, Korea). The primers used for PCR amplification are shown as follows: 5' CAGGCAGAGAATGCTGAGTTC-3', 5'-GATGTTGAG CGCAGGAACGCAGT-3' (ho-1), as well as 5'-GCCATGTACGTTGC ATC-3', 5'-CTCCTTAATGTCACGCAC-3' ( $\beta$-actin). Amplification products were resolved by $1.5 \%$ agarose gel electrophoresis, stained with safe dye and photographed by ImageQuant LAS 4000 .

\section{Antioxidant response element-luciferase reporter gene assay}

Cells were subcultured into 12 -well plates at a density of $5 \times$ $10^{4}$ cells per well prior to transfection. Cells were transfected with pARE-luc or pCMV- $\beta$-galactosidase using Genefectin transfection reagent (Genetrone Biotech, Gwangmyeong, Korea). The constructs for pARE-luc or pCMV- $\beta$-galactosidase were the kindly gift from Professor Young-Joon Surh (College of Pharmacy, Seoul National University, Seoul, Korea). Briefly, pARE-luc and pCMV- $\beta$-galactosidase were added to the optimem (serum free medium) containing Genefectin reagent and incubated for 20 minutes. After 24 hours of transfection, cells were treated with 
fraxetin $(50$ or $100 \mu \mathrm{M})$ for additional 6 hours and cell lysis was carried out with $\times 1$ reporter lysis buffer. After preparing cell lysates, the luciferase assay was performed as previously described. $^{24}$

\section{Preparation of cytosolic and nuclear extracts}

The cytosolic and nuclear extracts were prepared by using NEPER Nuclear and Cytoplasmic Extraction Reagent Kit (Thermo Scientific). Pellets were suspended in $50 \mu \mathrm{L}$ of CERI (Cytoplasmic Extraction Reagent I) for 15 minutes and added CERII (Cytoplasmic Extraction Reagent II) for additional 2 minutes. The mixture was centrifuged for 10 minutes at $16,000 \times g$. The supernatant is cytosolic extract. The pellets were washed with NER (Nuclear Extraction Reagent), incubated on ice for 1 hour and centrifuged at $16,000 \times g$ for 15 minutes. The supernatant is nuclear proteins. Prepared nuclear fraction was analyzed by Western blotting.

\section{Determination of reactive oxygen species production}

ROS generation was monitored by flow cytometry using the peroxide-sensitive fluorescent probe, DCF-DA. Briefly, cells were plated in 6-well culture plates at a density of $2 \times 10^{5}$ cells/well and treated with DMSO or fraxetin. After treatment, cells were incubated with $25 \mu \mathrm{M}$ DCF-DA in PBS at $37^{\circ} \mathrm{C}$ for 30 minutes, washed twice with HBSS solution, suspended in the complete media and examined under a fluorescence microscope or fluorescence activated cell sorter to detect the intracellular accumulation of ROS.

\section{Statistical analysis}

Data were expressed as mean \pm SD of three independent replicate experiments. Significant differences among groups were determined using Student's $t$-test. A value of $P<0.05$ was considered as statistically significant.

\section{RESULTS}

1. Effect of fraxetin on heme oxygenase-1 mRNA and protein expression in $\mathrm{HaCaT}$ cells

We first examined whether fraxetin induces any cytotoxicity in $\mathrm{HaCaT}$ cells by MTS assay. Incubation of HaCaT cells with fraxetin at indicated concentration did not affect the cell viability (Fig. 1B). Treatment of cells with fraxetin $(50$ or $100 \mu \mathrm{M})$ for 6 hours induced the expression of ho-1 mRNA (Fig. 1C) and protein (Fig. 1D) in a concentration-dependent manner. Moreover, treatment with fraxetin $(100 \mu \mathrm{M})$ for indicated time periods resulted in elevated expression of ho-1 mRNA (Fig. 1E) and protein (Fig. 1F) in a time-dependent manner.

2. Effect of fraxetin on nuclear factor-erythroid-2related factor-2 and antioxidant response element activation in $\mathrm{HaCaT}$ cells

Nrf2 is one of the transcription factors that regulates ho- 1 expression. Thus, we investigated whether Nrf2 is involved in fraxetin-induced HO-1 expression. As illustrated in Figure 2A, fraxetin treatment for 1 and 3 hours caused Nrf2 accumulation in the nucleus with corresponding decrease in cytosolic fraction. Since Nrf2 binds to the ARE sequences located in the promoter region of ho-1, we examined the effect of fraxetin on the ARE-

A
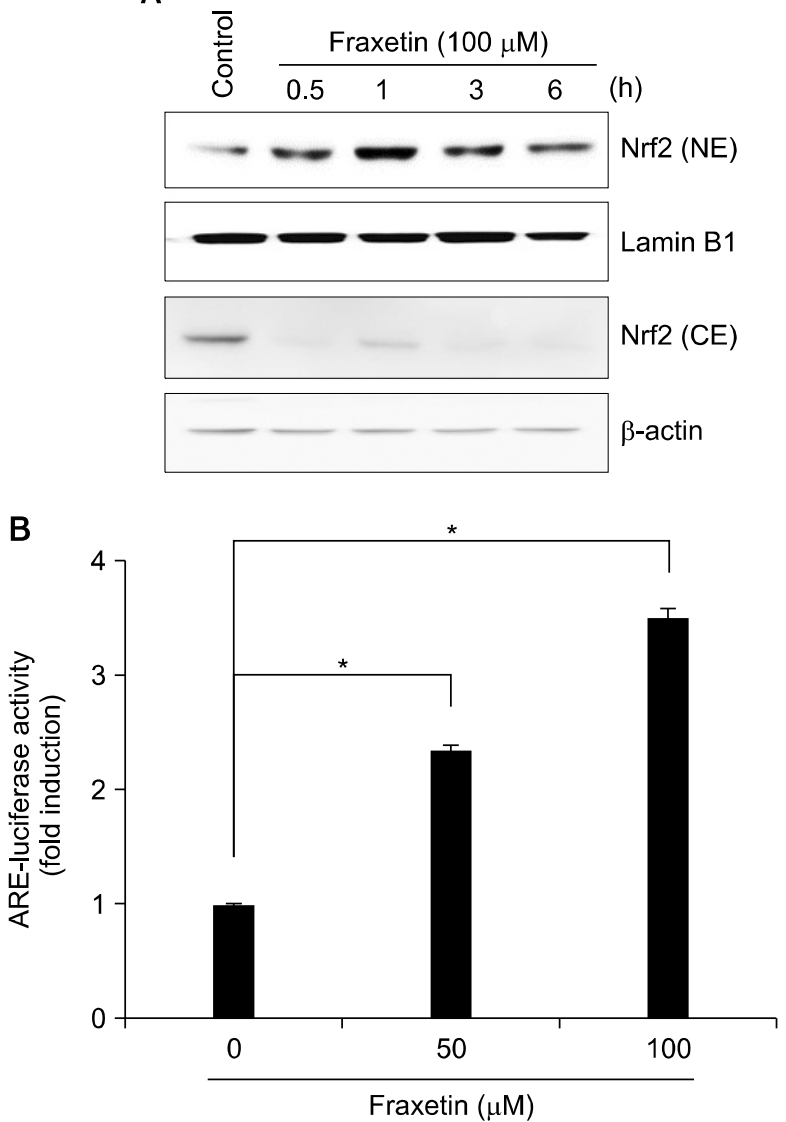

Figure 2. Effect of fraxetin on nuclear factor-erythroid-2-related factor-2 and antioxidant response element activation. (A) HaCaT cells were treated with fraxetin $(100 \mu \mathrm{M})$ for indicated periods and nuclear and cytosolic levels of nuclear factor-erythroid-2-related factor-2 (Nrf2) were determined by Western blot analysis. (B) Cells transiently transfected with the antioxidant response element (ARE)-luciferase construct or control vector were treated with fraxetin $(50$ and 100 $\mu \mathrm{M})$ for 6 hours and the ARE-luciferase activity was examined. The experiment was done in triplicate and the data are presented as mean $\pm \mathrm{SD}$. NE, nuclear extract; $\mathrm{CE}$, cytosolic extract. ${ }^{*} P<0.01$. 
reporter gene activity. Treatment with fraxetin increased the ARE luciferase activity in a concentration-dependent manner (Fig. 2B).

\section{Effect of fraxetin on phosphorylation of Akt and AMP-activated protein kinase $\alpha$ in $\mathrm{HaCaT}$ cells}

Activation of several upstream kinases has been reported to induce Nrf2 and subsequently increase the expression of HO-1. ${ }^{34,35}$ To explore the upstream signaling pathways involved in fraxetin-induced Nrf2 activation and HO-1 expression, we tested the effects of fraxetin on the activation of Akt and AMPK $\alpha$ in HaCaT cells. Treatment with fraxetin significantly increased the phosphorylation of Akt and AMPK $\alpha$ at 1 hour and 6 hours, respectively (Fig. 3A). Expression of PTEN, which is known as a negative regulator of Akt, was decreased at early time $(0.5,1$, and 3 hours) and then returned to the basal level at 6 hours in fraxetin-treated $\mathrm{HaCaT}$ cells (Fig. 3A). Next, we incubated HaCaT cells with LY294002 (a pharmacological inhibitor of Akt) and Compound C (an inhibitor of AMPK $\alpha$ ) for 1 hour before treatment with fraxetin and investigated the effect of these inhibitors on expression of HO-1. The fraxetin-induced expression of HO-1 was reversed by LY294002 or Compound C pretreatment (Fig. 3B and 3C). In our previous study, we found that thymoquinone induces HO-1 expression by Nrf2 activation through phosphorylation of Akt and AMPK $\alpha$ in HaCaT cells and Akt plays the role of an upstream kinase to AMPK $\alpha{ }^{24}$ Therefore, we investigated the possible cross regulation between fraxetin- induced phosphorylation of Akt and AMPK $\alpha$. However, in fraxetin-treated HaCaT cells, the pharmacological inhibition of Akt did not affect fraxetin-induced phosphorylation of AMPK $\alpha$, suggesting Akt is not involved in fraxetin-induced AMPK $\alpha$ activation (Fig. 3B).

\section{Role of Akt and AMP-activated protein kinase $\alpha$ in} fraxetin-induced nuclear factor-erythroid-2-related factor-2 activation in $\mathrm{HaCaT}$ cells

We then investigated the role of Akt or AMPK $\alpha$ in fraxetininduced Nrf2 activation. As illustrated in Figure 4, pharmacological inhibition of Akt and AMPK $\alpha$ attenuated fraxetin-induced nuclear localization of Nrf2, respectively. These results suggest that fraxetin induces HO-1 via activation of Akt/Nrf2 or $\mathrm{AMPK} \alpha / \mathrm{Nrf} 2$ pathway.

\section{Reactive oxygen species generation by fraxetin in HaCaT cells}

Cytoprotective proteins are induced as an adaptive response to mild oxidative stress as a safe guard against excessive ROSinduced cellular damage. ${ }^{4}$ Thus, a wide variety of natural
A

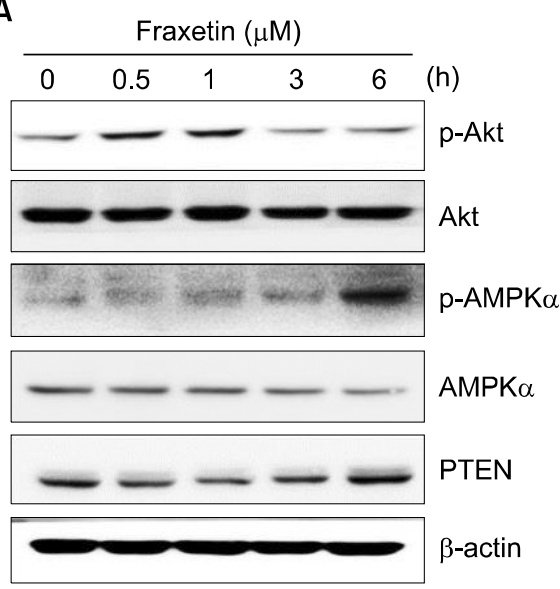

B

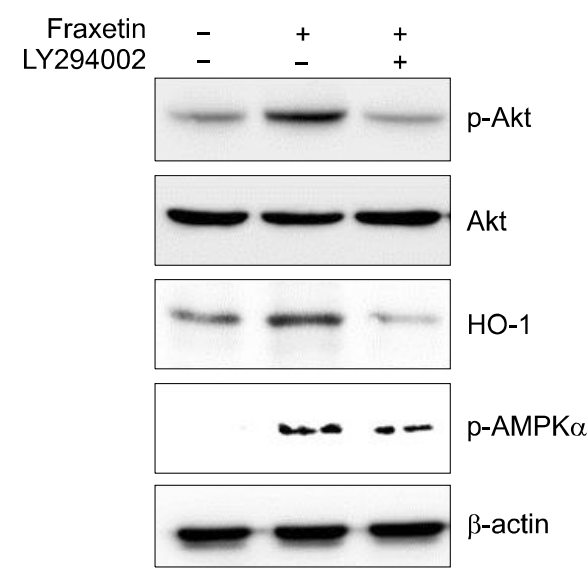

C

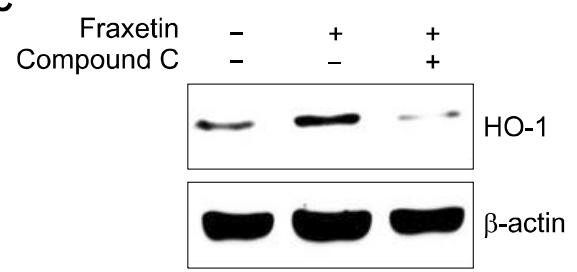

Figure 3. Effect of fraxetin on the activation of Akt and AMP-activated protein kinase $\alpha$ and expression of PTEN in HaCaT cells. (A) HaCaT cells were treated with fraxetin $(100 \mu \mathrm{M})$ for indicated periods. Cells were pretreated with (B) Akt inhibitor LY294002 (10 $\mu \mathrm{M})$ and $(\mathrm{C})$ AMP-activated protein kinase (AMPK) $\alpha$ inhibitor compound $\mathrm{C}(10 \mu \mathrm{M})$ for 1 hour prior to incubation with fraxetin (100 $\mu \mathrm{M})$ for 1 hour. Levels of Akt and heme oxygenase-1 (HO-1) were examined by Western blot analysis. PTEN, phosphatase and tensin homolog.

products capable of activating Nrf2 are shown to function as pro-oxidants capable of generating ROS. We examined whether fraxetin can generate ROS as a mechanism underlying its effect on Nrf2 activation and HO-1 expression. Treatment with fraxetin for 1 hour concentration-dependently increased ROS generation 
A

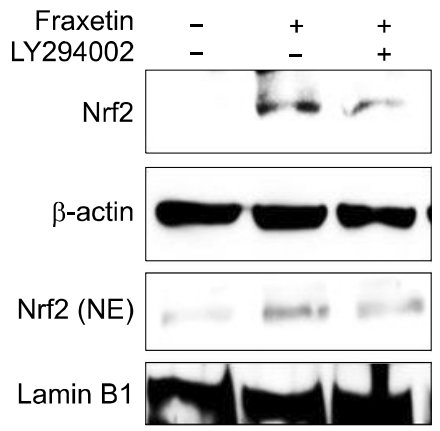

B

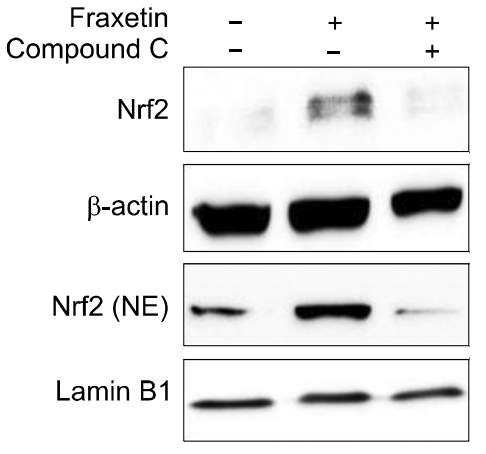

Figure 4. Roles of Akt and AMP-activated protein kinase $\alpha$ in fraxetin-induced nuclear factor-erythroid-2-related factor-2 activation and heme oxygenase-1 expression in HaCaT cells. Cells were pretreated with LY294002 (10 $\mu \mathrm{M})$ (A) and compound C (10 $\mu \mathrm{M})$ (B) for 1 hour prior to incubation with fraxetin $(100 \mu \mathrm{M})$ for 1 hour. Expression of nuclear factor-erythroid-2-related factor-2 (Nrf2) was examined by Western blot analysis. Data are representative of three different experiments. NE, nuclear extract.

A

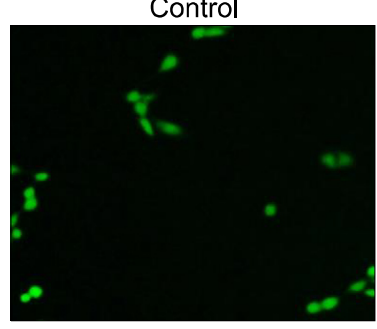

Fraxetin $50 \mu \mathrm{M}$

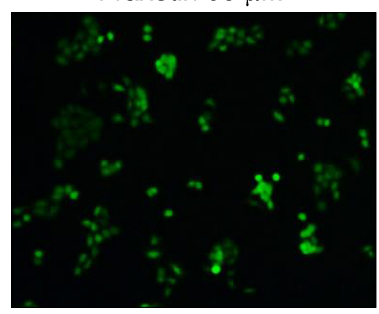

Fraxetin $100 \mu \mathrm{M}$

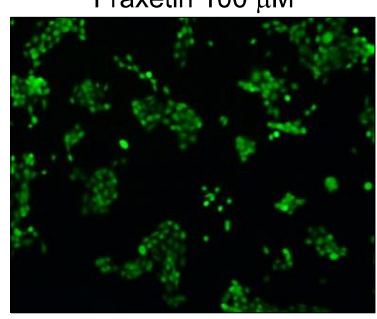

B

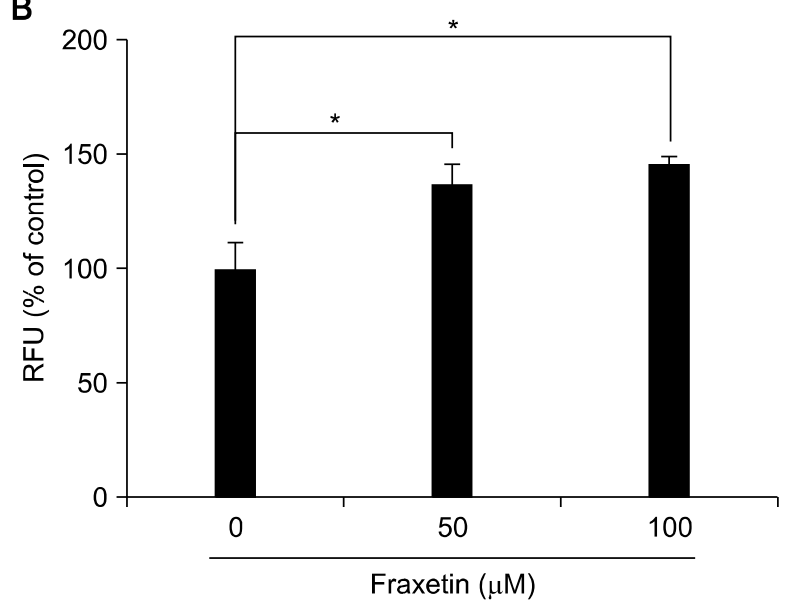

Figure 5. Effect of fraxetin on generation of reactive oxygen species in HaCaT cells. Cells were treated with indicated concentration of fraxetin for 1 hour and then examined for the intracellular accumulation of reactive oxygen species (ROS) (A) under the fluorescence microscope using $2^{\prime}$-,7'-dichlorofluorescin diacetate fluorescence staining method ( $\times$ 200) and (B) ELISA plate reader. The experiment was done in triplicate and the data are presented as mean \pm $\mathrm{SD}$. RFU, relative fluorescence unit. $* P<0.01$.

in $\mathrm{HaCaT}$ cells (Fig. 5). This finding suggests that fraxetin may function as a pro-oxidant that elicits an adaptive response to activate Nrf2 signaling and subsequent HO-1 expression in HaCaT cells.

\section{DISCUSSION}

The incidence of skin cancer is increasing worldwide. Ex- posure to solar UV radiation is the major etiologic factors for skin cancer. The generation of excessive ROS by UV induces oxidative modifications of cellular macromolecules, such as nucleic acids, proteins and lipids. ${ }^{36}$ ROS is eliminated by phase II detoxifying and antioxidant enzymes, such as HO-1, glutathioneS-transferase and $\mathrm{NAD}(\mathrm{P}) \mathrm{H}$ :quinone oxidoructase before they attack cellular macromolecules. ${ }^{37}$ Therefore, natural products capable of inducing cytoprotective proteins have been nominated 
as potential candidates for the chemoprevention of skin carcinogenesis. $^{4}$

Fraxetin is an O-methylated coumarin isolated from $F$ rhynchophylla. Fraxetin has been reported to exert antioxidative, ${ }^{28-30}$ anti-inflammatory, ${ }^{31}$ neuroprotective ${ }^{32}$ and antitumor $^{33}$ effects. For examples, fraxetin inhibits 5-lipoxygenase activity in polymorphonuclear leukocytes ${ }^{31}$ and also inhibits tumor growth and metastasis to the lung or liver in highly metastatic osteosarcoma LM8 cell-bearing mice. ${ }^{33}$ Furthermore, neuroprotective effect of fraxetin on rotenone-induced cytotoxicity also has been well demonstrated. ${ }^{38,39}$ However, any skin protective effect of fraxetin has not been investigated yet. Therefore, in this study, we examined the skin cytoprotective effect of fraxetin.

HO-1 is a stress response protein that catalyzes the degradation of heme to iron, billiverdin and carbon monoxide. Expression of HO-1 is highly induced by a wide variety of oxidants in many types of cells. ${ }^{40}$ The induction of HO-1 expression has been regarded as a crucial marker for oxidative stress, and it is involved in an adaptive protective response against oxidative damage. ${ }^{40}$ A recent study by Was et $a .^{8}$ demonstrated the protective role of HO-1 in mouse skin carcinogenesis. According to this study, HO-1 knock out mice are more susceptible to develop skin papillomas as compared with wild type animals. ${ }^{8}$ Shin et al. ${ }^{9}$ showed that overexpression of HO-1 remarkably decreases TPA-induced ROS production and transformation of JB6 cells. These data suggest the possible protective role of HO-1 in skin tumorigenesis. HO-1 expression is induced at the transcription level through the ARE located in the promoter region of Hmoxl which includes multiple copies of ARE sequences necessary for gene transcription by various inducers. ${ }^{41}$ These ARE sequences are binding sites for the Nrf2. Thus, Nrf2 acts as the major switch in transcriptional activation of ho-1 gene. ${ }^{11}$ Recent studies demonstrated that Nrf2 plays a pivotal role in the protection of the skin against UV irradiation in mouse fibroblasts, ${ }^{42}$ human skin fibroblasts ${ }^{43}$ and ketatinocytes. ${ }^{44}$ Therefore, Nrf2/ARE pathway activation protects the skin from UV-induced damage and has been suggested as an important therapeutic strategy for skin cancer. In this study, we demonstrated that fraxetin induces HO-1 expression and modulates Nrf2 signaling in HaCaT cells. This finding is in good agreement with the previous study which has demonstrated that fraxetin induces expression of HO-1 by activation of Nrf2 in vascular smooth muscle cells. ${ }^{25}$

In resting cells, Nrf2 resides in the cytoplasm by forming an inactive complex with its inhibitory protein Keap-1, which causes proteasomal degradation of Nrf2. Under mild oxidative condition, Nrf2 is liberated from Keap1 through oxidative or covalent modification of critical cysteine residues of Keap $1 .{ }^{13,16}$ On the other hand, Nrf2 can be dissociated from Keap1 upon phosphorylation at specific serine or threonine residues of Nrf2 by multiple upstream kinases, such as PI3K/Akt, ${ }^{19,45}$ and $\mathrm{AMPK}^{20}$ and MAP kinases. ${ }^{21}$ In our previous study, we found that thymoquinone, an active constituent of black cumin, induces HO-1 expression by activating Nrf2 through phosphorylation of AMPK $\alpha$ and Akt, which plays the role of an upstream kinase to AMPK $\alpha$ in HaCaT cells. ${ }^{24}$ In fraxetin-treated $\mathrm{HaCaT}$ cells, the compound also induced Nrf2 activation by phosphorylation of both Akt and AMPK $\alpha$. However, our study revealed that Akt is not involved in fraxetin-induced AMPK $\alpha$ activation. The reasons for these differences are unclear. Further studies are needed to investigate the possible crosstalk of these pathways in Nrf2/ARE activation by various phytochemicals.

Since HO-1 can be induced by various oxidative stresses, we speculated that ROS may be involved in fraxetin-induced HO-1 expression in $\mathrm{HaCaT}$ cells. As we expected, fraxetin induced ROS generation in a concentration-dependent manner. In our previous study, ROS played a critical role in HO-1 expression in HaCaT cells. ${ }^{24}$ These data suggest that the ability of fraxetin to induce HO-1 expression may be dependent on intracellular ROS production.

In conclusion, HaCaT cells incubated with fraxetin showed activation of Akt and AMPK $\alpha$, which appears to be responsible for nuclear translocation of Nrf2 and its subsequent binding to ARE, thereby upregulating HO-1 expression.

\section{ACKNOWLEDGMENTS}

This research was supported by Basic Science Research Program through the National Research Foundation of Korea (NRF) funded by the Ministry of Science, ICT and Future Planning (2014R1A2A2A01004698) and the Bio \& Medical Technology Development Program of the NRF funded by the Korean government, MSIP (2015M3A9B6073827). We also thank Dr. Joydeb Kumar Kundu for critical review of manuscript.

\section{CONFLICTS OF INTEREST}

No potential conflicts of interest were disclosed. 


\section{REFERENCES}

1. Duracková Z. Some current insights into oxidative stress. Physiol Res 2010;59:459-69.

2. Prawan A, Kundu JK, Surh YJ. Molecular basis of heme oxygenase-1 induction: implications for chemoprevention and chemoprotection. Antioxid Redox Signal 2005; 7:1688-703.

3. Taguchi K, Motohashi H, Yamamoto M. Molecular mechanisms of the Keap1-Nrf2 pathway in stress response and cancer evolution. Genes Cells 2011;16:123-40.

4. Chun KS, Kundu J, Kundu JK, Surh YJ. Targeting Nrf2-Keap1 signaling for chemoprevention of skin carcinogenesis with bioactive phytochemicals. Toxicol Lett 2014:229:73-84.

5. Kirino M, Kirino Y, Takeno M, Nagashima Y, Takahashi K, Kobayashi M, et al. Heme oxygenase 1 attenuates the development of atopic dermatitis-like lesions in mice: implications for human disease. J Allergy Clin Immunol 2008;122:290-7, 297.

6. Park KK, Park JH, Jung YJ, Chung WY. Inhibitory effects of chlorophyllin, hemin and tetrakis(4-benzoic acid)porphyrin on oxidative DNA damage and mouse skin inflammation induced by 12-O-tetradecanoylphorbol-13-acetate as a possible anti-tumor promoting mechanism. Mutat Res 2003:542:89-97.

7. Park JH, Lee CK, Hwang YS, Park KK, Chung WY. Hemin inhibits cyclooxygenase-2 expression through nuclear factor-kappa B activation and ornithine decarboxylase expression in 12-O-tetradecanoylphorbol-13-acetate-treated mouse skin. Mutat Res 2008;642: 68-73.

8. Was H, Sokolowska M, Sierpniowska A, Dominik P, Skrzypek K, Lackowska B, et al. Effects of heme oxygenase-1 on induction and development of chemically induced squamous cell carcinoma in mice. Free Radic Biol Med 2011;51:1717-26.

9. Shin JW, Ohnishi K, Murakami A, Lee JS, Kundu JK, Na HK, et al. Zerumbone induces heme oxygenase-1 expression in mouse skin and cultured murine epidermal cells through activation of Nrf2. Cancer Prev Res (Phila) 2011;4:860-70.

10. Khor TO, Huang MT, Kwon KH, Chan JY, Reddy BS, Kong AN. Nrf2-deficient mice have an increased susceptibility to dextran sulfate sodium-induced colitis. Cancer Res 2006;66:11580-4.

11. Alam J, Cook JL. Transcriptional regulation of the heme oxygenase-1 gene via the stress response element pathway. Curr Pharm Des 2003:9:2499-511.

12. Itoh $\mathrm{K}$, Wakabayashi N, Katoh $\mathrm{Y}$, Ishii T, Igarashi K, Engel JD, et al. Keap1 represses nuclear activation of antioxidant responsive elements by Nrf2 through binding to the amino-terminal Neh2 domain. Genes Dev 1999;13:76-86.

13. Dinkova-Kostova AT, Holtzclaw WD, Cole RN, Itoh K, Wakabayashi N, Katoh Y, et al. Direct evidence that sulfhydryl groups of Keap 1 are the sensors regulating induction of phase 2 enzymes that protect against carcinogens and oxidants. Proc Natl Acad Sci U S A 2002;99:11908-13.

14. Itoh $\mathrm{K}$, Wakabayashi N, Katoh $\mathrm{Y}$, Ishii $\mathrm{T}$, O'Connor T, Yamamoto M. Keap1 regulates both cytoplasmic-nuclear shuttling and degradation of Nrf2 in response to electrophiles. Genes Cells 2003; 8:379-91.

15. Kang MI, Kobayashi A, Wakabayashi N, Kim SG, Yamamoto M. Scaffolding of Keap1 to the actin cytoskeleton controls the function of Nrf2 as key regulator of cytoprotective phase 2 genes. Proc Natl Acad Sci U S A 2004;101:2046-51.
16. Wakabayashi N, Dinkova-Kostova AT, Holtzclaw WD, Kang MI, Kobayashi A, Yamamoto M, et al. Protection against electrophile and oxidant stress by induction of the phase 2 response: fate of cysteines of the Keap1 sensor modified by inducers. Proc Natl Acad Sci U S A 2004;101:2040-5.

17. Yu S, Khor TO, Cheung KL, Li W, Wu TY, Huang Y, et al. Nrf2 expression is regulated by epigenetic mechanisms in prostate cancer of TRAMP mice. PLoS One 2010;5:e8579.

18. Numazawa S, Ishikawa M, Yoshida A, Tanaka S, Yoshida T. Atypical protein kinase $\mathrm{C}$ mediates activation of NF-E2-related factor 2 in response to oxidative stress. Am J Physiol Cell Physiol 2003;285:C334-42.

19. Martin D, Rojo AI, Salinas M, Diaz R, Gallardo G, et al. Regulation of heme oxygenase-1 expression through the phosphatidylinositol 3-kinase/Akt pathway and the Nrf2 transcription factor in response to the antioxidant phytochemical carnosol. J Biol Chem 2004:279:8919-29.

20. Mo C, Wang L, Zhang J, Numazawa S, Tang H, Tang X, et al. The crosstalk between Nrf2 and AMPK signal pathways is important for the anti-inflammatory effect of berberine in LPS-stimulated macrophages and endotoxin-shocked mice. Antioxid Redox Signal 2014:20:574-88.

21. Yu R, Chen C, Mo YY, Hebbar V, Owuor ED, Tan TH, et al. Activation of mitogen-activated protein kinase pathways induces antioxidant response element-mediated gene expression via a Nrf2-dependent mechanism. J Biol Chem 2000;275:39907-13.

22. Cheung KL, Kong AN. Molecular targets of dietary phenethyl isothiocyanate and sulforaphane for cancer chemoprevention. AAPS J 2010;12:87-97.

23. Huang HC, Nguyen T, Pickett CB. Phosphorylation of Nrf2 at Ser-40 by protein kinase $C$ regulates antioxidant response element-mediated transcription. J Biol Chem 2002;277:42769-74.

24. Kundu J, Kim DH, Kundu JK, Chun KS. Thymoquinone induces heme oxygenase-1 expression in HaCaT cells via Nrf2/ARE activation: Akt and AMPK $\alpha$ as upstream targets. Food Chem Toxicol 2014;65:18-26.

25. Thuong PT, Pokharel YR, Lee MY, Kim SK, Bae K, Su ND, et al. Dual anti-oxidative effects of fraxetin isolated from Fraxinus rhinchophylla. Biol Pharm Bull 2009:32:1527-32.

26. Kim NY, Pae HO, Ko YS, Yoo JC, Choi BM, Jun CD, et al. In vitro inducible nitric oxide synthesis inhibitory active constituents from Fraxinus rhynchophylla. Planta Med 1999;65:656-8.

27. Jiang JH, Jin CM, Kim YC, Kim HS, Park WC, Park H. Anti-toxoplasmosis effects of oleuropein isolated from Fraxinus rhychophylla. Biol Pharm Bull 2008;31:2273-6.

28. Fernandez-Puntero B, Barroso I, Iglesias I, Benedí J, Villar A. Antioxidant activity of Fraxetin: in vivo and ex vivo parameters in normal situation versus induced stress. Biol Pharm Bull 2001:24:777-84.

29. Martín-Aragón S, Benedí JM, Villar AM. Modifications on antioxidant capacity and lipid peroxidation in mice under fraxetin treatment. J Pharm Pharmacol 1997;49:49-52.

30. Paya M, Goodwin PA, De Las Heras B, Hoult JR. Superoxide scavenging activity in leukocytes and absence of cellular toxicity of a series of coumarins. Biochem Pharmacol 1994:48:445-51.

31. Kimura Y, Okuda H, Arichi S, Baba K, Kozawa M. Inhibition of the formation of 5-hydroxy-6,8,11,14-eicosatetraenoic acid from arachidonic acid in polymorphonuclear leukocytes by various coumarins. Biochim Biophys Acta 1985;834:224-9. 
32. Molina-Jiménez MF, Sánchez-Reus MI, Andres D, Cascales M, Benedi J. Neuroprotective effect of fraxetin and myricetin against rotenone-induced apoptosis in neuroblastoma cells. Brain Res 2004;1009:9-16

33. Kimura Y, Sumiyoshi M. Antitumor and antimetastatic actions of dihydroxycoumarins (esculetin or fraxetin) through the inhibition of M2 macrophage differentiation in tumor-associated macrophages and/or G1 arrest in tumor cells. Eur J Pharmacol 2015:746:115-25.

34. Lee JS, Surh YJ. Nrf2 as a novel molecular target for chemoprevention. Cancer Lett 2005;224:171-84.

35. Surh YJ, Kundu JK, Na HK. Nrf2 as a master redox switch in turning on the cellular signaling involved in the induction of cytoprotective genes by some chemopreventive phytochemicals. Planta Med 2008;74:1526-39.

36. Sander CS, Chang H, Hamm F, Elsner P, Thiele JJ. Role of oxidative stress and the antioxidant network in cutaneous carcinogenesis. Int J Dermatol 2004:43:326-35.

37. Talalay P, Dinkova-Kostova AT, Holtzclaw WD. Importance of phase 2 gene regulation in protection against electrophile and reactive oxygen toxicity and carcinogenesis. Adv Enzyme Regul 2003:43:121-34.

38. Sánchez-Reus MI, Peinado II, Molina-Jiménez MF, Benedí J. Fraxetin prevents rotenone-induced apoptosis by induction of endogenous glutathione in human neuroblastoma cells. Neurosci Res 2005:53:48-56.
39. Molina-Jiménez MF, Sánchez-Reus MI, Cascales M, Andrés D, Benedí J. Effect of fraxetin on antioxidant defense and stress proteins in human neuroblastoma cell model of rotenone neurotoxicity. Comparative study with myricetin and N-acetylcysteine. Toxicol Appl Pharmacol 2005;209:214-25.

40. Tyrrell RM. Solar ultraviolet A radiation: an oxidizing skin carcinogen that activates heme oxygenase-1. Antioxid Redox Signal 2004:6:835-40.

41. Prestera T, Talalay P, Alam J, Ahn YI, Lee PJ, Choi AM. Parallel induction of heme oxygenase- 1 and chemoprotective phase 2 enzymes by electrophiles and antioxidants: regulation by upstream antioxidant-responsive elements (ARE). Mol Med 1995;1:827-37.

42. Hirota A, Kawachi Y, Itoh K, Nakamura Y, Xu X, Banno T, et al. Ultraviolet A irradiation induces NF-E2-related factor 2 activation in dermal fibroblasts: protective role in UVA-induced apoptosis. J Invest Dermatol 2005; 124:825-32.

43. Zhong JL, Edwards GP, Raval C, Li H, Tyrrell RM. The role of Nrf2 in ultraviolet A mediated heme oxygenase 1 induction in human skin fibroblasts. Photochem Photobiol Sci 2010;9:18-24.

44. Tian FF, Zhang FF, Lai XD, Wang LJ, Yang L, Wang X, et al. Nrf2-mediated protection against UVA radiation in human skin keratinocytes. Biosci Trends 2011:5:23-9.

45. Kim KC, Kang KA, Zhang R, Piao MJ, Kim GY, Kang MY, et al. Up-regulation of Nrf2-mediated heme oxygenase-1 expression by eckol, a phlorotannin compound, through activation of Erk and PI3K/Akt. Int J Biochem Cell Biol 2010;42:297-305. 